\title{
Evaluating a rigid-plastic method to estimate the earthquake ductility demand on structures
}

\author{
M. C. Porcu \& G. Carta \\ Dipartimento di Ingegneria Strutturale, University of Cagliari, Italy
}

\begin{abstract}
In order to evaluate the reliability of a rigid-plastic method in estimating the earthquake displacement ductility demand, the present paper applies the method to hundreds of different elastic-plastic oscillators under more than thirty recorded earthquakes. The mean ratio of the predicted value over the exact value of the displacement ductility demand is computed and plotted as a function of the vibration period of the oscillator for different values of the yield acceleration. The results show that, whatever the oscillator and the earthquake, the rigidplastic method leads to a generally conservative estimate of the inelastic displacement demand. Mean errors less than $15 \%$ are found both for comparatively short-period oscillators and for comparatively long-period oscillators. For medium-period oscillators, the relative mean error is generally less than $30 \%$, even for very high levels of ductility demand. Some advantages of the rigid-plastic method with respect to other approximate methods are also discussed in the paper.

Keywords: earthquake ductility demand, seismic inelastic displacement prediction, rigid-plastic method.
\end{abstract}

\section{Introduction}

The assessment of the earthquake ductility demand on structures is often carried out by means of approximate methods, most of which are based on the theory of linear elastic oscillators, cf. e.g. Rosemblueth and Herrera [1]; Gulkan and Sozen [2]; Iwan [3]; Kowalsky et al. [4]; Newmark and Hall [5]; Miranda [6].

An alternative method was proposed by Paglietti and Porcu [7] and subsequently improved by Porcu and Carta $[8,9]$, which predicts the maximum plastic displacement of an elastic-plastic oscillator from that of a rigid-plastic 
oscillator possessing the same ratio between yield strength and mass (yield acceleration). The peak displacement of the latter can be obtained from the earthquake rigid-plastic pseudo-spectrum, which is a single-curve response diagram (Paglietti and Porcu [7]; Domingues Costa et al [10]; Porcu and Mascia [11]). Section 2 recalls how the rigid-plastic method can be applied in practice.

To evaluate the reliability of such a method, the present paper analyses the results from hundreds of different elastic-plastic oscillators subjected to a significant variety of recorded ground motions. The mean ratio between estimated and calculated values of the displacement ductility demand is evaluated as a function of the period $T$ of the oscillator for different values of the yield acceleration. The results provided in Section 3 show that the rigid-plastic method is generally conservative. Moreover, it leads to small relative errors both in the short and in the long period range. Whereas in the medium period range the error may reach $30 \%$. In order to keep the errors of the rigid-plastic method below small percentage also in this range, an improvement of the empirical formula to be applied for medium period oscillator would be advisable. It should be noted, however, that these errors usually refer to high levels of ductility demand.

Some favourable features of the rigid-plastic method are finally discussed in Section 4. The method may be, in fact, faster to apply than other approximate methods available in current literature, which usually require iteration procedures to estimate the ductility demand on a structure. In addition, it singles out the range of periods in which the considered elastic-plastic oscillators may plastically yield under a given earthquake. This is a general result, which can be exploited by any method that aims to predict the earthquake inelastic displacement demand. Also when compared to other approximate methods, the rigid-plastic method is shown to provide good enough estimates, even when the plastic displacements are very large.

Other authors adopted a rigid-plastic approximation to model the response of ductile structures (e.g. Makris and Black [12]; Hibino et al. [13]). A rigid-plastic approach was also recently proposed by Domingues Costa et al. [14], which predicts the maximum plastic displacements of MDOF buildings by means of equivalent generalized SDOF systems. Thanks to this, the rigid-plastic method applied in the present paper could also be exploited to assess the seismic ductility demand of MDOF systems.

\section{The rigid-plastic method prediction}

The earthquake ductility demand of an elastic-perfectly-plastic oscillator can be defined by means of the following ratio:

$$
\mu=\frac{u_{\max }}{u_{y}},
$$

also referred to as the ductility factor or displacement ductility ratio (Chopra [15]). Here $u_{\max }$ is the absolute value of the maximum displacement of the 
oscillator, while $u_{y}$ is the absolute displacement at yield. When the elasticplastic oscillator deforms into the plastic range, $u_{\max }$ is greater than $u_{y}$ and, consequently, the ductility factor becomes greater than unity. In this case, $u_{\max }$ can be decomposed as follows:

$$
u_{\max }=u_{y}+u_{\max }^{P},
$$

where $u_{\max }^{P}$ denotes the absolute value of the maximum plastic displacement. The ductility ratio then becomes:

$$
\mu=1+\frac{u_{\max }^{P}}{u_{y}},
$$

On the other hand, the absolute displacement at yield $u_{y}$ is given by:

$$
u_{y}=\frac{F_{y}}{k}=\frac{T^{2}}{4 \pi^{2}} a_{y},
$$

$k, T, F_{y}$ and $a_{y}$ being the stiffness, the natural period of vibration, the yield strength and the yield acceleration of the oscillator, respectively. The yield acceleration is given by the ratio between the yield strength $F_{y}$ and the mass $M$ of the oscillator, and represents the maximum acceleration that the oscillator may reach during the motion (Chopra [15]; Paglietti and Porcu [7]). All displacements are here intended to be relative to the ground.

In view of eq. (4), the ductility factor can be also expressed as:

$$
\mu=1+\frac{4 \pi^{2}}{T^{2} a_{y}} u_{\text {max }}^{P}
$$

This equation shows that assessing the ductility demand for an elastic-plastic oscillator requires that $u_{\max }^{P}$ be known. For a given earthquake, $u_{\max }^{P}$ depends on the oscillator vibration period $T$, damping ratio $\xi$ and yield acceleration $a_{y}$. It should be calculated by numerical integration of the non-linear equations of motion of the elastic-plastic oscillator.

The rigid-plastic method proposed by Porcu and Carta [9] provides a simpler, though approximate, way to predict $u_{\max }^{P}$. It leads to the following formulae:

$$
\begin{array}{cl}
u_{\max }^{P}=u_{\max }^{R P} & \text { for } T \leq 0.13 T^{*}, \\
u_{\max }^{P}=u_{\max }^{R P}+\overline{\Delta u}^{p} & \text { for } 0.13 T^{*} \leq T \leq T^{*},
\end{array}
$$




$$
\begin{gathered}
u_{\max }^{P}=u_{\max }^{R P} \frac{T-\bar{T}}{T^{*}-\bar{T}} \quad \text { for } T^{*} \leq T \leq \bar{T}, \\
u_{\max }^{P}=0 \quad \text { for } T>\bar{T},
\end{gathered}
$$

where

$$
\overline{\Delta u} p=-0.039 a_{y}\left[\frac{2.5\left(\bar{T}+T^{*}\right)\left(1-\sqrt{\frac{a_{y}}{g}}\right)}{\sqrt[4]{\xi}}-\frac{\left(T^{*}\right)^{2}}{\bar{T}}-1\right]\left[\left(\frac{T}{T^{*}}\right)^{2}-1.13\left(\frac{T}{T^{*}}\right)+0.13\right] .
$$

In the above formula time should be expressed in seconds.

In equations (6a)-(6c) $u_{\max }^{R P}$ represents the maximum displacement that would be reached under the given earthquake by a rigid-plastic oscillator possessing the same yield acceleration as that of the elastic-plastic oscillator under consideration. For a given earthquake $u_{\max }^{R P}$ only depends on $a_{y}$, and can be calculated by integrating the non-linear equations of motion of the rigid-plastic oscillator, which are simpler than those of the elastic-plastic one (Paglietti and Porcu [7]). Alternatively, and more quickly, it can be obtained from the rigidplastic pseudo-spectrum of the earthquake, (cf. Paglietti and Porcu [7]; Porcu and Mascia [11]; Domingues Costa et al. [10]). An instance of how obtaining $u_{\max }^{R P}$ from a rigid-plastic pseudo-spectrum, for a given value of $a_{y}$, is provided in Figure 1.

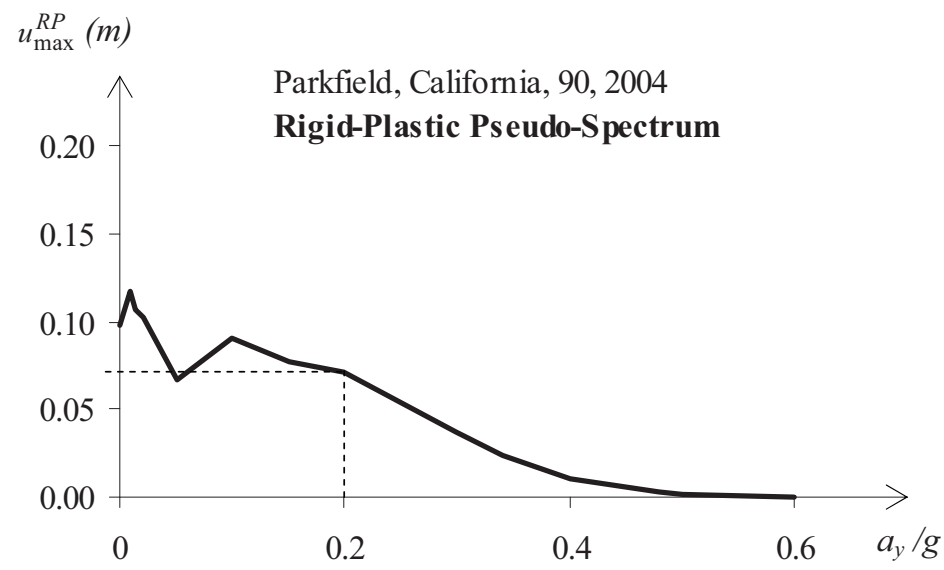

Figure 1: Obtaining $u_{\max }^{R P}$ from the earthquake rigid-plastic pseudo-spectrum. 
Quantities $T^{*}$ and $\bar{T}$ appearing in eqns (6) and (7) are two characteristic values of period that depend on the earthquake and are function of $a_{y}$ and $\xi$. They can easily be obtained by intercepting the displacement elastic response spectrum with the following curves (Porcu and Carta [8]):

$$
\begin{gathered}
u^{*}\left(T, a_{y}\right)=\frac{a_{y} T^{2}}{4 \pi^{2}} \sqrt{\frac{8 \pi^{2} u_{\max }^{R P}}{a_{y} T^{2}}+1}, \\
u_{y}\left(T, a_{y}\right)=\frac{a_{y} T^{2}}{4 \pi^{2}}
\end{gathered}
$$

Figure 2 shows how obtaining $T^{*}$ and $\bar{T}$ from an elastic response spectrum.

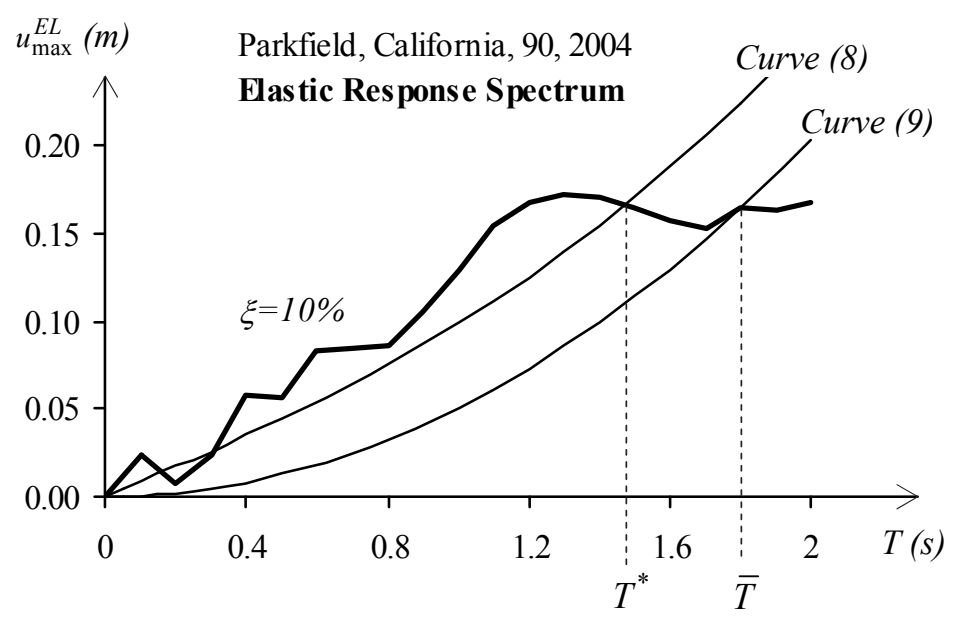

Figure 2: Determining $T^{*}$ and $\bar{T}$ from the elastic displacement response spectrum (for $a_{y}=0.2 \mathrm{~g}$ ).

Once that $u_{\max }^{R P}$ is taken from the rigid-plastic pseudo-spectrum and the pair of characteristic periods $T^{*}$ and $\bar{T}$ are found from the elastic response spectrum, $u_{\max }^{p}$ can be predicted directly from eqns (6) and (7). An example on how to get such a prediction is given in Figure 3 .

By introducing the estimated value of $u_{\max }^{p}$ into eqn (5), the ductility factor $\mu$ is finally obtained. For the same instance considered in Figures 1-3, Figure 4 plots both the predicted and the "exact" values of the ductility factor $\mu$. Figure 4 shows that $\mu$ tends to unity as $T$ tends to $\bar{T}$. Indeed, for $T=\bar{T}$ it is $u_{\max }^{p}=0$ and, therefore, $\mu=1$, as follows from eqn (5). According to eqn (5), 
moreover, for any given value of $a_{y}$ the ductility factor tends to infinity as $T$ tends to zero. This makes a direct comparison between predicted and "exact" values of $\mu$ inaccurate in the short-period range. For this reason, the ratio between predicted and exact values of $\mu$ will instead be considered in the next section, which allows for a comparison in the whole range of periods.

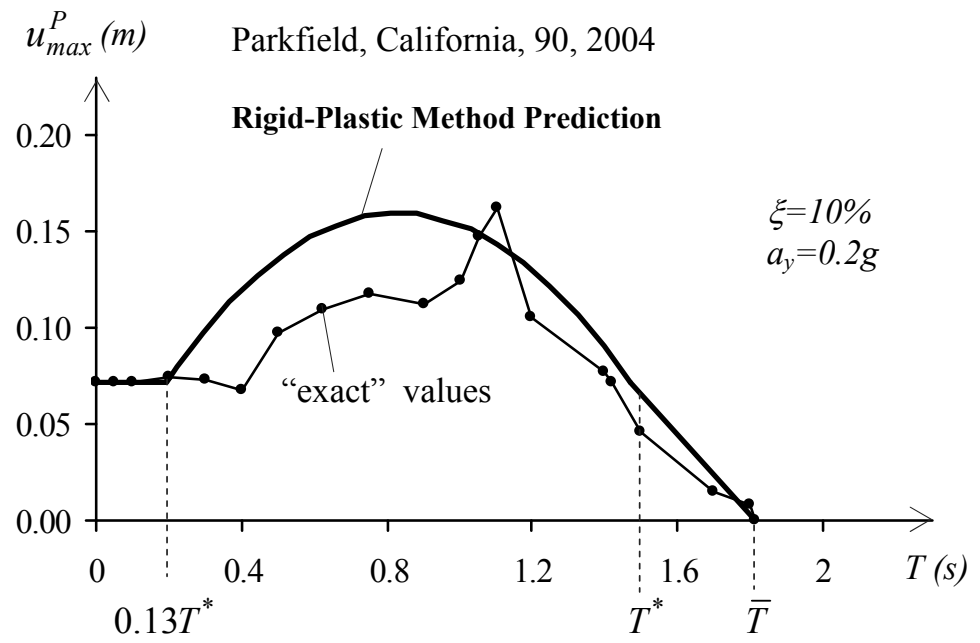

Figure 3: Predicting the peak plastic displacements through the rigid-plastic method.

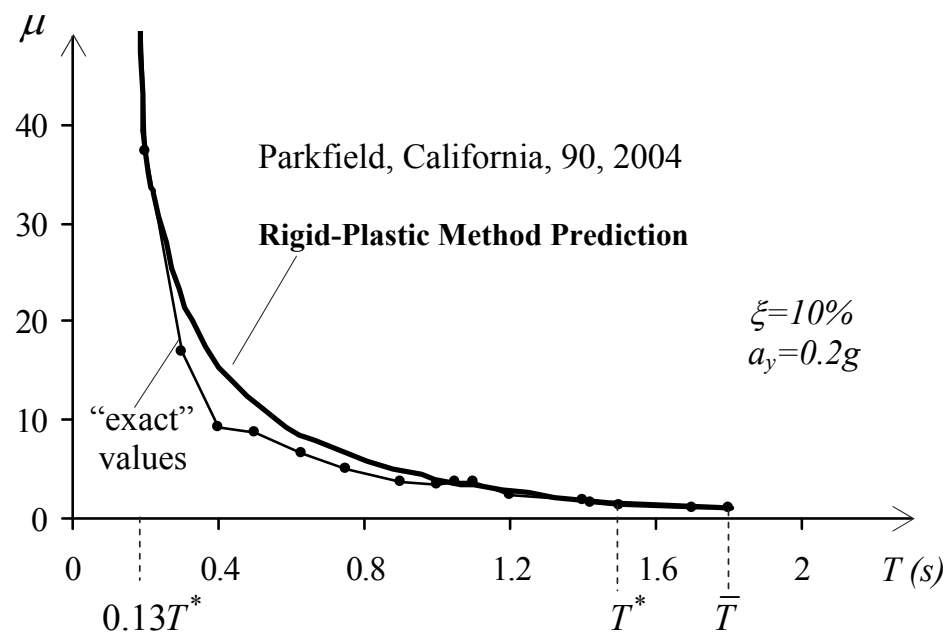

Figure 4: Predicting the ductility factor $\mu$ by means of the rigid-plastic method. 


\section{Mean relative errors in the rigid-plastic prediction}

In order to evaluate the relative errors that can be made when estimating the earthquake ductility demand by means of the rigid-plastic method, the ratio between the earthquake displacement ductility factor as estimated by means of the rigid-plastic method, say $\tilde{\mu}$, and the "exact" value $\mu$ of the same factor computed by a non-linear time-history analysis will be considered below. In view of eq. (3), this ratio can be expressed as:

$$
r=\frac{\tilde{\mu}}{\mu}=\frac{u_{y}+\tilde{u}_{\max }^{P}}{u_{y}+u_{\max }^{P}},
$$

where $\tilde{u}_{\max }^{P}$ indicates the plastic displacement estimated through eqns (6) and (7) and $u_{\max }^{P}$ the calculated "exact" value. The ratio $r$ gives the relative error we introduce when estimating the ductility factor $\mu$ with the rigid-plastic method. A comparison between eqn (10) and eqn (3) shows that $r$ also gives the ratio between the estimated and the calculated total displacements.

As the estimated and the calculated values of the plastic displacement tend to coincide, the ratio $r$ tends to unity, which means that no error is committed in estimating the ductility demand, or, similarly, the total displacement. This obviously happens if $T$ equals zero (rigid-plastic behaviour), and also when $T$ is equal to $T^{*}$, since in both these cases $u_{\max }^{p}=\tilde{u}_{\max }^{P}$. On the other hand, $r$ is equal to unity also when $T \geq \bar{T}$, since it is $u_{\max }^{p}=\tilde{u}_{\max }^{P} \equiv 0$. In all these cases, the rigid-plastic method predicts the earthquake displacement demand exactly. Otherwise, some errors can be produced.

Table 1: $\quad$ Earthquakes considered in the present investigation.

\begin{tabular}{|l|l|c|l|}
\hline 1 & Ardal (Iran), LONG, 1977 & 17 & Landers (California), LCN000, 1992 \\
\hline 2 & Cape Mendocino (Cal), PET090, 1992 & 18 & Loma Prieta (Cal), CLS000, 1989 \\
\hline 3 & Cartago (Costa Rica), LONG, 1991 & 19 & Mammoth Lakes(Cal), LLUL000, 1999 \\
\hline 4 & Chamoli (India), N20E, 1999 & 20 & Montenegro, N-S, 1979 \\
\hline 5 & Chi Chi (Taiwan), CHY041N, 1999 & 21 & Morgan Hill (Cal), CYC195, 1984 \\
\hline 6 & Coalinga (California), D-TSM360, 1983 & 22 & N. Palm Springs (Cal), NPS300, 1986 \\
\hline 7 & Duzce (Turkey), DZC270, 1999 & 23 & Parkfield (California), C02065, 1966 \\
\hline 8 & Edgecumbe (New Zealand), N07W, 1987 & 24 & Parkfield (California), 90, 2004 \\
\hline 9 & El Salvador, LONG, 2001 & 25 & San Fernando (California), S16E, 1971 \\
\hline 10 & Erzincan (Turkey), N279, 1992 & 26 & South Iceland, LONG, 2000 \\
\hline 11 & Friuli (Italy), E-W, 1976 & 27 & Spitak (Armenia), GUK000, 1988 \\
\hline 12 & Gazli (Uzbekistan), E-W, 1976 & 28 & Superstitn Hills (Cal), B-SUP135, 1987 \\
\hline 13 & Imperial Valley (Cal), H-BCR230, 1979 & 29 & Tabas (Iran), N74E, 1978 \\
\hline 14 & Irpinia (Italy), A-STU270, 1980 & 30 & Tabas (Iran), TAB-LN, 1978 \\
\hline 15 & Kobe (Japan), N35W, 1995 & 31 & Trinidad, B-RDE000, 1980 \\
\hline 16 & Kocaeli (Turkey), ATS000, 1999 & 32 & Victoria (Mexico), CPE045, 1980 \\
\hline
\end{tabular}


To evaluate the extent of these errors, a numerical investigation was carried out. By referring to the earthquakes listed in Table 1, the value of the ratio $r$ as defined by eqn (10) was computed for elastic-plastic oscillators possessing different realistic values of $a_{y}$ and a damping ratio $\xi=10 \%$. The latter is a typical value for damping ratio when stress is at the yield point, cf. Chopra [15]. For each earthquake and for each value of $a_{y}$, different values of natural period, ranging from zero to $\bar{T}$, were considered.

For each value of $T$, the mean value of the ratio $r$, say $M_{r}$, was finally obtained. The resulting diagram is presented in Figure 5. It shows that, whatever the yield acceleration and whatever the natural period $T$ of the elastic-plastic oscillator, the rigid-plastic method does on average provide a conservative estimate of the displacement ductility factor (values of $M_{r}$ larger than one). In particular, the mean relative errors are generally lower than $30 \%$. They are very low for $T<0.2 s$ and always lower than $15 \%$ for $T>0.75 s$.

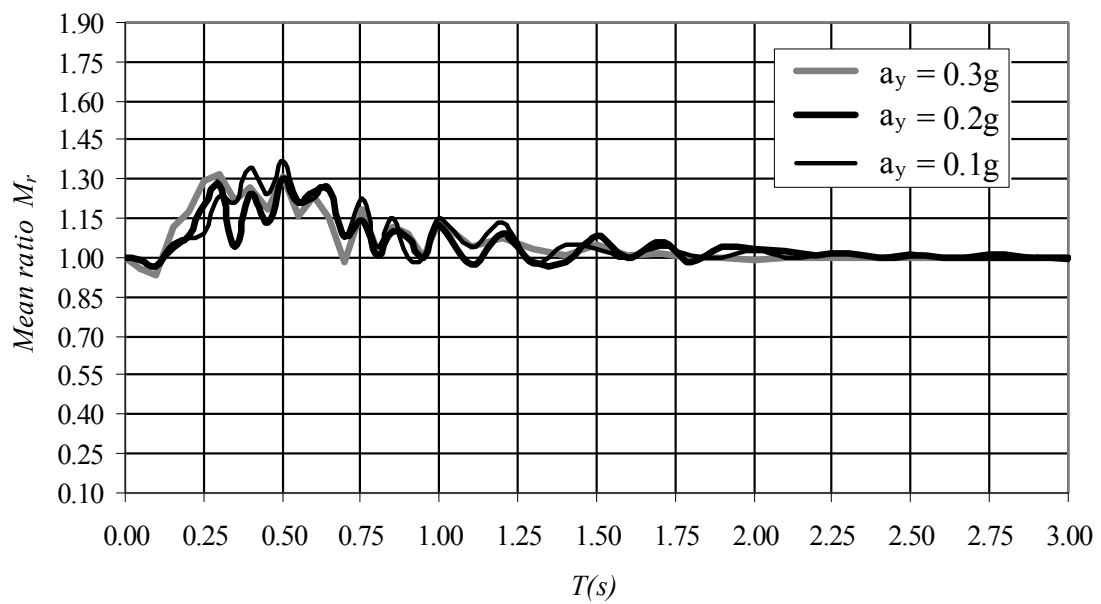

Figure 5: Mean ratio of predicted to calculated maximum displacements for different values of $a_{y}(\xi=10 \%)$.

\section{Evaluating the rigid-plastic method}

Most of the approximate methods proposed in current literature estimate the earthquake ductility demand $\mu$ by means of parameters that are function of $\mu$ itself. As a consequence, they usually require iteration procedures which may also imply convergence problems, cf. e.g. Chopra and Goel [16]; Miranda and Akkar [17]. An in-depth evaluation of the accuracy of some of these methods was done by Miranda and Ruiz Garcia [18] and by Akkar and Miranda [19]. 
On the contrary, the rigid-plastic method does not involve any iteration procedure. Should the rigid-plastic pseudo-spectrum and the elastic response spectrum of the considered earthquake be available, the method predicts the inelastic displacement demand on any elastic-plastic oscillator by means of a direct procedure, see Section 2.

What is more, the rigid-plastic method spots the range of periods in which the inelastic demand prediction actually needs to be obtained under a given earthquake. This range is always given by $0 \leq T \leq \bar{T}$. The actual value of $\bar{T}$, which is different for different earthquakes and for different values of $a_{y}$ and $\xi$, may be easily obtained from the elastic response spectrum of the considered earthquake, see Figure 2. General as it is, this result could be adopted by any approximate method that aims at estimating the earthquake ductility demand on elastic-plastic oscillators.

Within the above range, the rigid-plastic method gives a good estimate of the peak plastic displacement of comparatively short-period oscillators (say $T \leq 0.25 s$ ) and comparatively long-period oscillators $(T>0.75 s)$, see Figure 5. On the contrary, high errors are generally encountered in the short period range when other approximate methods are adopted, as can be inferred from the diagrams presented by Miranda and Ruiz Garcia [18] and by Akkar and Miranda [19]. For $T>0.75 \mathrm{~s}$ the rigid-plastic method prediction is on average comparable with that of other approximate methods (cf. e.g. the results presented by Miranda and Ruiz Garcia [18] and by Akkar and Miranda [19]).

In the medium period range the rigid-plastic prediction may be, however, less satisfactory. Here the empirical formula (7) plays a fundamental part for the rigid-plastic prediction. Most of the mean errors found in the range $0.25 \mathrm{~s} \leq T \leq 0.75 \mathrm{~s}$ (see Figure 5) can be actually put down to the appliance of such a formula. It should be noted that in the same range of periods some other approximate methods might give a better prediction on average (cf. Miranda and Ruiz Garcia [18]; Akkar and Miranda [19]). This could denote the need for an improvement of formula (7).

The following points should be noted, though. The mean ratio $M_{r}$ plotted in Figure 5 is relevant to assigned values of $a_{y}$, which may entail very high values of $\mu$ in the short-medium period range ( $\mu>10)$, as Figure 4 shows. This means that the rigid-plastic method is able to estimate very high values of $\mu$ with reasonably narrow mean errors. On the contrary, rather low values of $\mu$ are usually assigned when evaluating the mean errors relevant to other approximate methods (for example $\mu$ ranging from 2 to 6 is considered by Miranda and Ruiz Garcia [18]). In addition, the errors relevant to these methods generally increase as $\mu$ increases, this being especially so in the medium period range (cf. Miranda and Ruiz Garcia [18]; Akkar and Miranda [19]). This means that also in the medium period range the rigid-plastic prediction can in fact be good enough, even when compared to that obtained by means of other approximate methods. 


\section{Conclusions}

With the aim of evaluating the extent of the relative errors that can be made when the maximum seismic displacement of an elastic-plastic oscillator is estimated by means of the rigid-plastic method, a numerical investigation was carried out in the present paper. The results show that this method leads -on average- to conservative and fairly good predictions, whatever the oscillator and the earthquake, and even when the plastic displacements are very large. Mean relative errors lower than $15 \%$ are found both for short-period and long-period oscillators. For medium period oscillator the error is generally less than $30 \%$. A profitable feature of the method is that of obtaining the ductility demand prediction directly in the range of periods where inelastic displacements may actually occur under a given earthquake. Moreover, the rigid-plastic prediction does not involve any iterative procedure, as many other approximate methods do. For short-period oscillators the mean error is generally much lower than that relevant to most of the other approximate methods. For medium-period and longperiod oscillators, a comparable mean error may be found with respect to other methods. Especially as the ductility demand increases.

\section{Acknowledgements}

The recorded accelerograms considered in this paper were obtained from: ESD - The European Strong-Motion Database, http://www.isesd.cv.ic.ac.uk PEER Strong Motion Database, http://peer.berkeley.edu/smcat COSMOS Virtual Data Center, http://db.cosmos-eq.org

\section{References}

[1] Rosemblueth, E. \& Herrera, I., On a kind of hysteretic damping. Journal of Engineering Mechanics Division ASCE, 90, pp. 37-48, 1964.

[2] Gulkan, P. \& Sozen, M., Inelastic response of reinforced concrete structures to earthquakes motion. ACI Journal, 71, pp. 604-610, 1974.

[3] Iwan, W.D., Estimating inelastic response spectra from elastic spectra. Earthquake Engineering and Structural Dynamics, 8, pp. 375-388, 1980.

[4] Kowalsky, M., Priestley, M.J.N. \& McRae, G.A., Displacement-based design of RC bridge columns in seismic regions. Earthquake Engineering and Structural Dynamics, 24, pp. 1623-1643, 1995.

[5] Newmark, N.M., \& Hall, W.J., Earthquake Spectra and Design, Earthquake Engineering Research Institute: Berkeley, CA, 1982.

[6] Miranda, E., Inelastic displacements ratios for structures on firm sites. Journal of Structural Engineering, 126, pp. 1150-1159, 2000.

[7] Paglietti, A. \& Porcu, M.C., Rigid-plastic approximation to predict motion under strong earthquakes. Earthquake Engineering and Structural Dynamics, 30, pp. 115-126, 2001. 
[8] Porcu, M.C. \& Carta, G., Rigid-plastic bound to the seismic inelastic response of flexible elastic-plastic oscillators. European Earthquake Engineering, 3, pp. 3-9, 2007.

[9] Porcu, M.C. \& Carta, G., Rigid-plastic seismic analysis to predict the structural ductility demand. International Journal of Applied Engineering Research, 4(3), pp. 309-325, 2009.

[10] Domingues Costa, J.L., Bento, R., Levtchitch, V. \& Nielsen, M.P., Simplified non-linear time-history analysis based on the Theory of Plasticity. Proc. of the ERES 2005 Conference, WIT Press: Southampton, UK, pp. 375-386, 2005.

[11] Porcu, M.C. \& Mascia, M., Rigid-plastic pseudo-spectra: peak response charts for seismic design. European Earthquake Engineering, 3, pp. 37-47, 2006.

[12] Makris, N. \& Black, C.J., Dimensional analysis of rigid-plastic structures under pulse-type excitations. Journal of Engineering Mechanics, 130(9), pp. 1006-1018, 2004.

[13] Hibino, Y., Toshikatsu, I., Domingues Costa, J.L. \& Nielsen, M.P., Procedure to predict the storey where plastic drift dominates in two-storey building under strong ground motion. Earthquake Engineering and Structural Dynamics, 38(7), pp. 929-939, 2008.

[14] Domingues Costa, J.L., Bento, R., Levtchitch, V. \& Nielsen, M.P., Rigidplastic seismic design of reinforced concrete structures. Earthquake Engineering and Structural Dynamics, 36, pp. 55-76, 2007.

[15] Chopra, A.K., Dynamics of Structures. Theory and Application to Earthquake Engineering, Prentice Hall: New Jersey, 2001.

[16] Chopra, A.K. \& Goel, R.K., Evaluation of NSP to estimate seismic deformation: SDF systems. Journal of Structural Engineering, 126(4), pp. 482-490, 2000.

[17] Miranda, E. \& Akkar, S.D., Evaluation of iterative schemes in equivalent linear methods. Earthquake Engineering Research Institute, 2003.

[18] Miranda, E. \& Ruiz Garcia, J., Evaluation of approximate methods to estimate maximum inelastic displacement demands. Earthquake Engineering and Structural Dynamics, 31, pp. 539-560, 2002.

[19] Akkar, S.D. \& Miranda, E., Statistical evaluation of approximate methods for estimating maximum deformation demands on existing structures. Journal of Structural Engineering, 131, pp. 160-172, 2005. 